\title{
Electromagnetic Modes in Deformed Nuclei
}

\author{
J. Kvasil, R.G. Nazmitdinov ${ }^{1}$, A. Mackova , M. Kopal \\ Department of Nuclear Physics, Charles University, 18000 Praha 8, Czech Republic \\ N. Lo Iudice \\ Università di Napoli "Federico II" and INFN I-80125 Napoli, Italy \\ V.O. Nesterenko \\ 1 Bogoliubov Laboratory of Theoretical Physics, JINR, 141980 Dubna, Russia \\ A strength function method is adopted to describe a coupling between \\ electric and magnetic modes of different multipolarity. The collective vibra- \\ tions are analysed for a separable residual interaction in the framework of \\ the random-phase approximation. The coupling between $M 2$ and $E 1$ giant \\ resonances is considered as an illustrative example.
}

PACS number(s): 21.10.R, 21.60.E, 21.60.J

\section{INTRODUCTION}

The probe of nuclear structure via electromagnetic interactions provides important information on the nuclear wave function and dynamics of nucleon-nucleon interactions. The nuclear response upon different external fields is measured by means of electromagnetic transitions between different quantum states. The transition amplitude at the emission (absorption) of a photon of a given multipolarity is proportional to matrix elements of multipole moments. Since in nuclear photo-processes the wave length $\lambda \approx 1 / k$ of the photon is larger then the nucleus radius $R$ (the long-wave approximation $k R<<1$ ) the multipole moments take the form [1]

$$
\begin{aligned}
\mathcal{M}(E \lambda \mu)= & \sum_{i=1}^{A} e_{\text {eff }}^{(\lambda)}(i) r_{i}^{\lambda} Y_{\lambda \mu}\left(\hat{r}_{i}\right) \\
\mathcal{M}(M \lambda \mu)= & \frac{\mu_{N}}{2} \sqrt{\lambda(\lambda+1)} \sum_{i=1}^{A}\left(g_{S, e f f}^{(\lambda)}(i)\left[\sigma_{i} \otimes Y_{\lambda-1}\left(\hat{r}_{i}\right)\right]_{\lambda \mu}\left(\vec{\sigma}_{(i)} \cdot \vec{Y}_{\lambda \mu}^{\lambda-1}\left(\hat{r}_{(i)}\right)\right)\right. \\
& \left.\left.+g_{l, e f f}^{(\lambda)}(i) \frac{4}{\lambda+1}\left[l_{i} \otimes Y_{\lambda-1}\left(\hat{r}_{i}\right)\right]_{\lambda \mu}\right] \cdot\left(\vec{l}_{(i)} \cdot \vec{Y}_{\lambda \mu}^{\lambda-1}\left(\hat{r}_{(i)}\right)\right)\right) r_{(i)}^{\lambda-1}
\end{aligned}
$$


where $e_{\text {eff }}^{(\lambda)}(i)$ is the nucleon effective charge of multipolarity $\lambda, g_{S, \text { eff }}^{(\lambda)}(i)$ and $g_{l, e f f}^{(\lambda)}(i)$ are the corresponding effective spin and orbital gyromagnetic ratios, respectively, and $\mu_{N}=\frac{e \hbar}{2 m c}$ is the nuclear magneton. In this approximation the total decay probability from the quantum state $\mid i>$ to the quantum state $\mid f>$ is determined as

$$
\begin{aligned}
T_{i \rightarrow f}\left(E_{\gamma}\right)= & 5.49985 * 10^{22} \sum_{\lambda}\left[\frac{E_{\gamma}}{(197.327)}\right]^{2 \lambda+1} \frac{2 \lambda+1}{\lambda[(2 \lambda+1) ! !]^{2}} \\
& {[B(E \lambda, i \rightarrow f)+0.011064 * B(M \lambda, i \rightarrow f)] }
\end{aligned}
$$

where the reduced transition probability for the electromagnetic transition of type $X$ $(X=E$ or $M)$ and multipolarity $\lambda$ is defined as

$$
B\left(X \lambda ; I_{i} K_{i}^{\pi_{i}} \nu_{i} \rightarrow I_{f} K_{f}^{\pi_{f}} \nu_{f}\right)=\frac{1}{2 I_{i}+1}\left|\left\langle I_{f} K_{f} \nu_{f}\|\mathcal{M}(X \lambda)\| I_{i} K_{i} \nu_{i}\right\rangle\right|^{2}
$$

Here the $B(E \lambda, i \rightarrow f)$ is given in $e^{2} f m^{2 \lambda}, B(M \lambda, i \rightarrow f)$ is given in $\mu_{N}^{2} f m^{2 \lambda-2}$, the transition energy $E_{\gamma}$ is defined in $M e V$ and the total decay probability $T_{i \rightarrow f}\left(E_{\gamma}\right)$ is given in $1 / s$.

At high excitation energy the density of nuclear states becomes so large that the description in terms of transition probabilities between individual states loses its practical sense. Strength function techniques are known to represent a very valuable tool for studying the electromagnetic properties of nuclei in energy regions with high level density [2] [4]. For example, defining the strength function as

$$
S\left(X \lambda, E_{\gamma}\right)=\sum_{\nu} B(X \lambda, g r \rightarrow \nu) \delta\left(E_{\gamma}-\omega_{\nu}\right)
$$

we can describe the distribution of strength of the electromagnetic excitation of the ground state over a range of the available excitation energy $E_{\gamma}$. Consequently, the strength function can be used for the analysis of the photo-absorption cross section $\sigma\left(E_{\gamma}\right)$

$$
\begin{aligned}
\sigma\left(E_{\gamma}\right)= & 1.40534 * 10^{5} \sum_{\lambda}\left[\frac{E_{\gamma}}{(197.327)}\right]^{2 \lambda-1} \frac{\lambda+1}{\lambda[(2 \lambda+1) ! !]^{2}} \\
& {[S(E \lambda, i \rightarrow f)+0.011064 * S(M \lambda, i \rightarrow f)] }
\end{aligned}
$$

Here, $S(E \lambda, i \rightarrow f), S(M \lambda, i \rightarrow f)$ are given in $\frac{e^{2} f m^{2 \lambda}}{M e V}, \frac{\mu_{N}^{2} f m^{2 \lambda-2}}{M e V}$, respectively. The comparison of the strength functions extracted from photo-absorption experiments with theoretical 
estimates can testify the basic model assumptions used for the calculations. Notice, that in the expression Eq.(5) there is a restriction due to only the parity selection rules. Therefore, such excitations contain contributions from different electric and magnetic operators which are able to create the excitation with a given parity.

In axially deformed nuclei rotational bands are built on the intrinsic states characterized by the angular momentum projection $K$ and the parity. In fact, such intrinsic states can be created by different electric and magnetic operators with all possible multipolarities in the nuclear Hamiltonian. However, there is a restriction on the parity quantum number again. For example, the state with $K^{\pi}=1^{-}$can be created by $E 1, M 2, E 3, M 4 \ldots$ operators. In standard approaches the coupling between different electric and magnetic modes allowed by the parity selection rules is neglected [2.5], however, there is no justification for this assumption. Based on the strength function method [3], we propose the generalisation of this approach [4] which allows consistently treat the contribution of different electromagnetic modes to the strength of excitations (de -excitations), in particular, in region of giant resonances.

\section{THE MODEL}

For the analysis of collective and single-particle degrees freedom of nucleus we use the the phonon+rotor model described in details in [4]. The model Hamiltonian is

$$
H=H_{s p}+H_{\text {pair }}+H_{\text {res }}+H_{\text {rot }}
$$

where $H_{s p}$ is a spherical one-body Hamiltonian (a spherical Nilsson model), $H_{\text {pair }}$ represents the pairing residual interaction, $H_{\text {res }}$ stands for the consistent with the one-body Hamiltonian the long-range residual interaction and $H_{\text {rot }}$ is the Hamiltonian of the rotor [2]. We use the ansatz of separable multipole-multipole and spin-multipole-spin-multipole forces including the isoscalar and isovector components

$$
H_{\text {res }}=-\frac{1}{2} \sum_{\lambda \mu} \sum_{\tau=0,1} \kappa_{\lambda}[\tau] M_{\lambda \mu}^{\dagger}[\tau] M_{\lambda \mu}[\tau]-\frac{1}{2} \sum_{l \lambda \mu} \sum_{\tau=0,1} \kappa_{l \lambda}[\tau] S_{l \lambda \mu}^{\dagger}[\tau] S_{l \lambda \mu}[\tau]
$$


Here $\kappa_{\lambda}[\tau], \kappa_{l \lambda}[\tau]$ are the multipole strength constants and

$$
\begin{aligned}
& M_{\lambda \mu}\left[\begin{array}{l}
0 \\
1
\end{array}\right]=M_{\lambda \mu}^{(p)} \pm M_{\lambda \mu}^{(n)}, \\
& S_{l \lambda \mu}\left[\begin{array}{l}
0 \\
1
\end{array}\right]=S_{l \lambda \mu}^{(p)} \pm S_{l \lambda \mu}^{(n)},
\end{aligned}
$$

are isoscalar $(\tau=0)$ and isovector $(\tau=1)$ multipole fields, which are obtained from proton and neutron multipole operators of the form:

$$
\begin{aligned}
M_{\lambda \mu}^{(\tau)} & =\sum_{\substack{q_{1} \sigma_{1} \in \tau \\
q_{2} \sigma_{2} \in \tau}}\left\langle q_{1} \sigma_{1}\left|R_{\lambda}(r) Y_{\lambda \mu}(\hat{r})\right| q_{2} \sigma_{2}\right\rangle a_{q_{1} \sigma_{1}}^{\dagger} a_{q_{2} \sigma_{2}}, \\
S_{l \lambda \mu}^{(\tau)} & =\sum_{\substack{q_{1} \sigma_{1} \in \tau \\
q_{2} \sigma_{2} \in \tau}}\left\langle q_{1} \sigma_{1}\left|R_{l \lambda}(r)\left[\sigma \otimes Y_{l}(\hat{r})\right]_{\lambda \mu}\right| q_{2} \sigma_{2}\right\rangle a_{q_{1} \sigma_{1}}^{\dagger} a_{q_{2} \sigma_{2}},
\end{aligned}
$$

For symmetry reasons it is convenient to construct multipole fields of a good signature

$$
R_{1}^{-1} F[r] R_{1}=r F[r]
$$

where $r= \pm 1$ and $R_{1} \equiv e^{i \pi \hat{J}_{x}}$. The details about the symmetry properties of the operators can be found in [4]. After performing the signature transformation, the Hamiltonian Eq.(6) can be expressed through the quasiparticle creation (annihilation) $\alpha_{q \sigma}^{\dagger}\left(\alpha_{q \sigma}\right)$ operators

$$
H=<H F B|H| H F B>+\sum_{k} \varepsilon_{q} \sum_{\sigma} \alpha_{q \sigma}^{\dagger} \alpha_{q \sigma}+H_{\text {pair }}+H_{\text {res }}+H_{\text {rot }}
$$

in the Hartree-Fock-Bogoliubov approximation. Here $\varepsilon_{q}$ is the quasiparticle energy. The pairing interaction $H_{\text {pair }}$ and the long-range residual interaction $H_{\text {res }}$ expressed in terms of the quasiparticle operators consist of terms proportional to combinations of type $\alpha^{\dagger} \alpha^{\dagger} \alpha^{\dagger} \alpha^{\dagger}$, $\alpha^{\dagger} \alpha^{\dagger} \alpha^{\dagger} \alpha, \alpha^{\dagger} \alpha^{\dagger} \alpha \alpha$ and h.c.. At the description of even-even nuclei the terms proportional to $\alpha^{\dagger} \alpha^{\dagger} \alpha^{\dagger} \alpha$ and h.c. are not considered, since they create (annihilate) the odd number of particles. The terms proportional to $\alpha^{\dagger} \alpha^{\dagger} \alpha^{\dagger} \alpha^{\dagger}$ and h.c are usually neglected in the description of the vibrational states in the harmonic approximation (see e.g. [2.5]). The remaining terms proportional to $\alpha^{\dagger} \alpha^{\dagger} \alpha \alpha$ are treated in the random phase approximation (RPA). After solution of the RPA equation of motion for collective modes [3] the Hamiltonian Eq.(11] can be expressed in terms of the RPA creation and annihilation phonons $\mathcal{Q}_{\nu}^{\dagger}, \mathcal{Q}_{\nu}$ 


$$
H_{R P A}=\sum_{\substack{\nu \\ \omega_{\nu} \neq 0}} \omega_{\nu}\left(\mathcal{Q}_{\nu}^{\dagger} \mathcal{Q}_{\nu}+\frac{1}{2}\right)+\frac{1}{2} \sum_{\substack{\nu_{0} \\ \omega_{\nu_{0}}=0}} \mathcal{P}_{\nu_{0}}^{2}
$$

and the corresponding phonon energies $\omega_{\nu}$. The last term in Eq.(12) represents the contribution of the spurious modes, for example, related to the rotation and translation in the coordinate space or to the rotation in the isospin space [3,5]. The RPA phonon defined by the collective coordinate $\mathcal{X}_{\nu}$ and momentum $\mathcal{P}_{\nu}$ has the following two-quasiparticle structure

$$
\mathcal{Q}_{\nu}^{\dagger}=\frac{1}{\sqrt{2}}\left[\sqrt{\omega_{\nu}} \mathcal{X}_{\nu}-\frac{i}{\sqrt{\omega_{\nu}}} \mathcal{P}_{\nu}\right]=\sum_{k l}\left(\psi_{k \hat{l}}^{\nu} \alpha_{k \hat{l}}^{\dagger} \alpha_{k \hat{l}}^{\dagger}-\varphi_{k \hat{l}}^{\nu} \alpha_{\hat{l} k} \alpha_{\hat{l} k}\right)
$$

Notice that Eq.(13) is valid only for the positive signature phonons which consist of the twoquasiparticle states $\alpha_{k \hat{l}}^{\dagger} \alpha_{k \hat{l}}^{\dagger}$. A similar expression can be written for the negative signature phonons built from the two-quasiparticle states $\alpha_{k l}^{\dagger} \alpha_{k l}^{\dagger}$ and $\alpha_{\hat{k} \hat{l}}^{\dagger} \alpha_{\hat{k} \hat{l}}^{\dagger}$. Since in the phonon+rotor model the rotation is treated adiabatically, there is a degeneracy with respect to the quantum number of signature and all physical properties can be described within the space of the positive signature states only 4 . In this approximation the wave function of the system can be written in the following form

$$
\left|\nu I M K^{\pi}\right\rangle=\sqrt{\frac{2 I+1}{16 \pi^{2}\left(1+\delta_{K 0}\right)}}\left\{\mathcal{D}_{M K}^{I}(\theta)\left|\psi_{\nu \pi K}\right\rangle+(-1)^{I} \mathcal{D}_{M-K}^{I}(\theta) R_{1}\left|\psi_{\nu \pi K}\right\rangle\right\}
$$

where $\left|\psi_{\nu \pi K}\right\rangle$ is the intrinsic wave function determined by one-phonon or multi-phonon states. The solution of the RPA equation of motion leads to the system of algebraic equations which defines the characteristic equation [3, 14. The size of this equation is determined by the number of the operators involved in the residual interaction. It is clear that the phonon energies being the roots of this equation and the structure of the two-quasiparticle amplitudes $\psi^{\nu}$ and $\varphi^{\nu}$ are dependent on the type of the residual interaction. In the other words, the presence or absence of the coupling between the electric and magnetic modes should influence on the structure of the RPA solutions. 


\section{DISCUSSION}

In order to avoid the cumbersome procedure of finding all phonon energies $\omega_{\nu}$ needed for the determination of the strength function $S\left(X \lambda, E_{\gamma}\right)$, Eq.(四), we developed the averaging technique [3]. The advantage of this procedure consist of the avoiding: i)the resolving the many-dimensional characteristic equation for each single root $\omega_{\nu}$ and ii)calculations of corresponding eigenvectors of the Hamiltonian and the $B\left(X \lambda, \omega_{\nu}\right)$. We consider the averaged strength function

$$
\mathcal{S}_{\Delta}(\omega)=\sum_{\nu} B(\omega) \rho_{\Delta}\left(\omega-\omega_{\nu}\right)
$$

with the averaging function such

$$
\rho_{\Delta}(x)=\frac{\Delta}{2 \pi} \frac{1}{x^{2}+(\Delta / 2)^{2}}, \quad \int_{-\infty}^{+\infty} d x \rho_{\Delta}(x)=1, \quad \lim _{\Delta \rightarrow 0} \rho_{\Delta}(x)=\delta(x)
$$

In Ref. [6] it was shown that the results are fairly independent of the choice of the averaging function. Considering the function Eq.(15) as a complex function with a complex argument, we apply the Cauchy theorem and finally obtain the following result

$$
\begin{aligned}
& \mathcal{S}_{\Delta}\left(X ; \nu_{g r} I_{i}=K_{i}=0 \rightarrow K^{\pi} I ; \omega\right)=-\left.\frac{2}{\pi} \operatorname{Im} \frac{\operatorname{det}(\hat{B}(z))}{\operatorname{det}(\hat{D}(z))}\right|_{z=\omega+i \frac{\Delta}{2}}+ \\
& \frac{\Delta}{2 \pi} \sum_{i j}\left(p_{i j}^{X I K}\right)^{2}\left[\frac{1}{\left[\left(\varepsilon_{i}+\varepsilon_{j}\right)-\omega\right]^{2}+\frac{\Delta^{2}}{4}}-\frac{1}{\left[\left(\varepsilon_{i}+\varepsilon_{j}\right)+\omega\right]^{2}+\frac{\Delta^{2}}{4}}\right]
\end{aligned}
$$

Here $D$ is the characteristic determinant of the RPA algebraic equations and $p_{i \hat{j}}^{X I K}$ is a quasiparticle matrix element of the transition operator. The matrix $B$ is similar to the matrix $D$, however, it has the dimension by 1 greater than that of the matrix $D$

$$
\begin{aligned}
B_{11}(\omega)=0, B_{i 1}(\omega) & =-B_{1 i}(\omega), \quad(i=2, \ldots, n) \\
B_{i j}(\omega) & =D_{i-1, j-1}(\omega),(i, j=2, \ldots, n)
\end{aligned}
$$

The first column and the first row of the matrix $B$ contain sums of the product of quasiparticle matrix elements of the transition operator and of the operators of the residual interaction (see details in [4]). The first term in Eq.(17) is related to the residual interaction and the second term is determined by the mean field. 
As the example of the coupling of different multipoles and spin-multipoles in the Hamiltonian we consider the $M 2$ strength function $\mathcal{S}_{\Delta}(M 2, E)=\sum_{K} \mathcal{S}_{\Delta}\left(M 2, E ; g r \rightarrow 2^{-} K\right)$ for ${ }^{154} \mathrm{Sm}$. In Fig. 1 the solid line corresponds to the coupling of $M_{1 K}$ (electric dipole) and $S_{12 K}$ (magnetic quadrupole) operators. The dashed line represents the contribution of the magnetic quadrupole term alone. The contribution of the plain quasiparticle term (the second term in Eq.(17)) is shown with the dotted line. Whereas the residual interaction changes the distribution of the strength of the magnetic quadrupole transitions, the influence of the electric dipole mode to the total strength function is relatively small. It seems that in the region of giant resonances the coupling between electric and magnetic operators for the M2 mode is essentially not important. The analysis of this coupling for low-lying states and for the other operators is in progress.

[1] A. Bohr and B.R. Mottelson, Nuclear Structure, V.1 (Benjamin, New York, 1969)

[2] V.G. Soloviev, Theory of Atomic Nuclei: Quasiparticles and Phonons (Institute of Physics, Bristol, 1992)

[3] J. Kvasil and R.G. Nazmitdinov, Sov. J. Part. Nucl. 17, 265 (1986)

[4] J. Kvasil, N. Lo Iuduce, V.O. Nesterenko and M. Kopal, Phys.Rev. C58, 209 (1998)

[5] P. Ring and P. Schuck, The Nuclear-Many Body Problem (Springer, New York, 1980)

[6] L.A. Malov, Preprint JINR, P4-1-228, 1981.

Figure Capture

Fig.1 The total $M 2$ strength function vs the excitation energy $E$. 


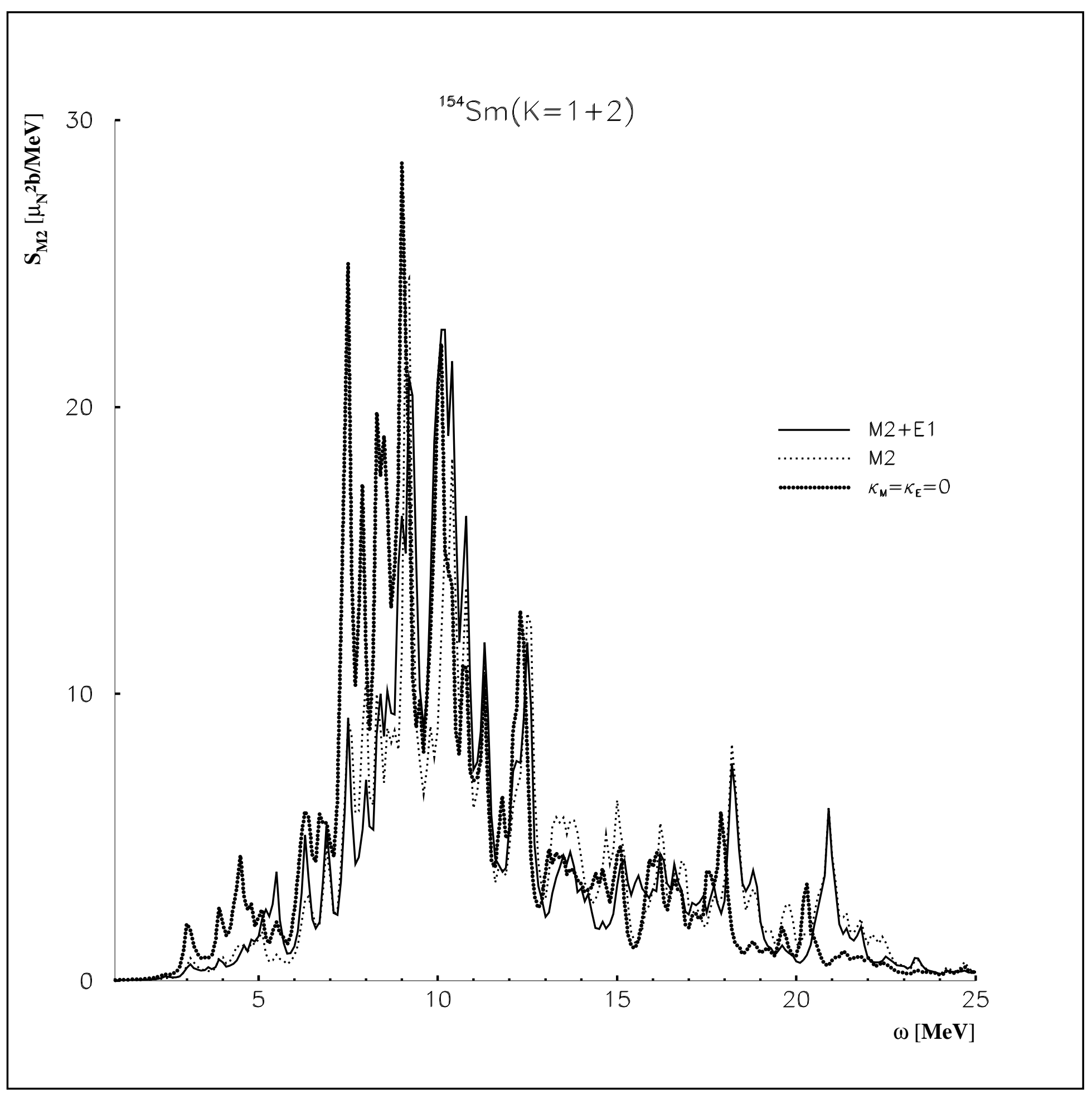

